\title{
Hospital-level intracranial pressure monitoring utilization and functional outcome in severe traumatic brain injury: a post hoc analysis of prospective multicenter observational study
}

\author{
Tomoya Okazaki* (D, Kenya Kawakita and Yasuhiro Kuroda
}

\begin{abstract}
Background: Several observational studies have shown that hospital-level intracranial pressure (ICP) monitoring utilization varies considerably in patients with severe traumatic brain injury (TBI). However, the relationship between hospital-level ICP monitoring utilization and clinical functional outcomes is unknown. This study examined whether patients with severe TBI treated at hospitals with high ICP monitoring utilization have better functional outcomes.

Methods: A post hoc analysis of the data from a prospective multicenter cohort study in Japan was undertaken, and included severe TBI patients (Glasgow Come Scale score $\leq 8$ ). The primary exposure was hospital-level ICP monitoring utilization. Patients treated at hospitals with more than $80 \%$ ICP monitoring utilization were assigned to a high group and the others to a low group. The primary endpoint was a favorable functional outcome at 6 months after injury, defined as a Glasgow Outcome Scale score of good recovery or moderate disability. We conducted multiple logistic regression analyses adjusted for potential confounders.

Results: Of the 427 included patients, 60 were assigned to the high group and 367 to the low group. Multiple logistic regression analysis revealed that patients in the high group had significantly better functional outcome (adjusted odds ratio [OR]: 2.36; 95\% confidence interval [Cl]: 1.17-4.76; $p=0.016$ ). Multiple logistic regression analysis adjusted for additional confounders supported this result (adjusted OR: 2.30; 95\% Cl: 1.07-4.92; $p=0.033$ ).

Conclusion: Treatment at hospitals with high ICP monitoring utilization for severe TBI patients could be associated with better functional outcome.

Keywords: Traumatic brain injury, Hospital-level intracranial pressure monitoring utilization, Patient-level intracranial pressure monitoring utilization, Functional outcome
\end{abstract}

* Correspondence: tomoyaokazaki4028@gmail.com

Emergency Medical Center, Kagawa University Hospital, 1750-1 Ikenobe, Kita,

Miki, Kagawa 761-0793, Japan

(c) The Author(s). 2021 Open Access This article is licensed under a Creative Commons Attribution 4.0 International License, which permits use, sharing, adaptation, distribution and reproduction in any medium or format, as long as you give appropriate credit to the original author(s) and the source, provide a link to the Creative Commons licence, and indicate if changes were made. The images or other third party material in this article are included in the article's Creative Commons licence, unless indicated otherwise in a credit line to the material. If material is not included in the article's Creative Commons licence and your intended use is not permitted by statutory regulation or exceeds the permitted use, you will need to obtain permission directly from the copyright holder. To view a copy of this licence, visit http://creativecommons.org/licenses/by/4.0/. The Creative Commons Public Domain Dedication waiver (http://creativecommons.org/publicdomain/zero/1.0/) applies to the data made available in this article, unless otherwise stated in a credit line to the data. 


\section{Background}

Traumatic brain injury (TBI) is a leading cause of trauma-related death or disability and confers a serious social burden worldwide [1]. Severe TBI, usually defined on the basis of a Glasgow Coma Scale (GCS) score of less than or equal to 8 , is especially associated with high mortality and unfavorable functional outcomes [2].

Elevated intracranial pressure (ICP) commonly occurs during the acute phase of TBI management and is both, a cause and consequence of secondary brain injury that leads to unfavorable functional outcomes [3, 4]. ICP monitoring is significantly associated with reduced short- and long-term mortality [5, 6]; however, there is insufficient evidence that the utilization of patient-level ICP monitoring can contribute to improved functional outcomes $[7,8]$.

Several observational studies have shown that hospitallevel ICP monitoring utilization for severe TBI varies dramatically $[5,9,10]$ and ranges from 9.6 to $65.2 \%$, as reported in a study from Los Angeles [9]. Another study from Japan reported utilization in the range of 0 to $100 \%$ [10]. However, the relationship between hospitallevel ICP monitoring utilization and clinical outcomes is controversial $[5,9]$.

This study was conducted to examine whether severe TBI patients treated at hospitals with high ICP monitoring utilization have better functional outcomes. The study utilized data from the Japan Neurotrauma Data Bank (JNTDB) Project 2015.

\section{Materials and methods}

\section{Study design and setting}

This study involved a post hoc analysis of data from the JNTDB Project 2015, a prospective multicenter cohort study that was conducted in 32 hospitals in Japan between April 2015 and March 2017. All of the participating institutions had a neurosurgical department and neurosurgeons who were actively involved in the management of TBI patients. The protocol was approved by the Institutional Review Board of each hospital, and all patients or their proxies provided written informed consent. The JNTDB Project 2015 enrolled TBI patients with a GCS score of 8 or lower within $48 \mathrm{~h}$ after injury or those requiring craniotomy, regardless of their GCS score. Study participants were followed up until hospital discharge or 6 months after the injury. The details of JNTDB Project 2015 and other JNTDB projects have been described in previous reports [11-13].

\section{Selection of participants}

For the purposes of this study, we included adult patients with severe TBI (GCS score $\leq 8$, age $\geq 18$ years) without treatment restrictions and an Abbreviated Injury Scale score of 6 , and excluded those with missing data for the ICP monitoring utilization, Glasgow Outcome Scale (GOS) score at 6 months after injury, age, GCS score on admission, pupillary reflex, hypotension on admission (systolic blood pressure $<90 \mathrm{mmHg}$ ), Marshall Computed Tomography (CT) classification, Injury Severity Score (ISS), and hospital type (university or non-university). Moreover, patients treated at hospitals with fewer than 5 patients were excluded because of the hospital-level analyses designed for this study.

\section{Data collection}

The following data were collected for analysis: the ICP monitoring use, hospital type, age, sex, prescription for anticoagulant drugs, prescription for antiplatelet drugs, GCS on admission, pupillary reflex (none, one, or both), hypotension on admission, body temperature on admission, Marshall CT classification, Abbreviated Injury Scale score, ISS, isolated TBI (defined as any Abbreviated Injury Scale score of 0 , other than that for the head), cause of injury, and 6-month post-injury GOS score.

\section{Exposure}

The primary exposure was the hospital-level ICP monitoring utilization, which was calculated as the ratio of the patients treated with ICP monitoring to the total number of patients who met the criteria for this study at each hospital. We classified hospitals into two categories based on their ICP monitoring utilization: patients treated at hospitals with more than and less than $80 \%$ ICP monitoring utilization were assigned to a "high" and a "low" group, respectively. The $80 \%$ cutoff was based on previous studies in the United States which found that, between 2007 and 2013, nearly 80\% of severe TBI patients were treated with ICP monitoring $[14,15]$.

The secondary exposure was the patient-level ICP monitoring utilization. Patients treated with and without ICP monitoring were assigned to an "ICP (+)"and an "ICP (-)" group, respectively.

\section{Outcome measures}

The primary endpoint was a favorable functional outcome at 6 months after injury, defined as a GOS score of 4 or 5 (moderate disability or good recovery) [16]. The other three categories based on the GOS score were: 1, death; 2, persistent vegetative state; and 3 , severe disability.

\section{Statistical analysis}

Continuous variables were analyzed using the MannWhitney $U$ test, and categorical comparisons were conducted with the Fisher's exact or chi-square test.

To clarify selection biases, we initially compared the baseline characteristics of patients included into the final analysis and those who were excluded due to missing data or fewer cases. Thereafter, we calculated the hospital- 
level ICP monitoring utilization, and compared the baseline characteristics and functional outcome at 6 months after injury in the high and low groups. We conducted a multiple logistic regression analysis that was adjusted for age, GCS motor score, and pupillary reflex to examine the association of hospital-level ICP monitoring utilization with the primary outcome (model 1). The abovementioned factors were selected on the basis of the International Mission for Prognosis and Analysis of Clinical Trials in TBI (IMPACT) Core model [17-19]. We performed another multiple logistic regression analysis adjusted for the age, GCS motor score, pupillary reflex, hypotension on admission, Marshall CT classification, and ISS, as well as the hospital type as a hospital-level variable (model 2). These factors were determined by referring to the IMPACT Extended model [17-19] and clinical plausible.

Furthermore, to evaluate the validity of the threshold between the high and the low group, we conducted sensitivity analysis based on further subclassification of the hospital-level ICP monitoring utilization. We ranked hospitals into five categories based on their ICP monitoring utilization rates $(0-19 \%, 20-39 \%, 40-59 \%, 60-$ $79 \%$, and $80-100 \%)$ and conducted multiple logistic regression analyses adjusted for the variables of the model 1 and model 2 .

To examine a possible association between the secondary exposure and the primary outcome, we compared the baseline characteristics and functional outcome at 6 months after injury between the ICP $(+)$ and the ICP (-) group. After adjusting for the variables of the model 1 and model 2, we conducted multiple logistic regression analyses.

A two-sided $P$-value less than 0.05 was considered statistically significant. Missing data were not replaced or estimated. All statistical analyses were conducted in EZR (Saitama Medical Center, Jichi Medical University, Saitama, Japan) [20].

\section{Results}

The JNTDB Project 2015 enrolled 1345 patients during the study period. Of the 662 potential participants, 427 (65\%) met the eligibility criteria for this study (Fig. 1). A comparison of the baseline characteristics between the patients included in the final analysis and those who were excluded are shown in Additional Table 1. The ICP monitoring utilization at each hospital remarkably ranged from 0 to $100 \%$ (Fig. 2).

Sixty patients (14\%) who were treated at four hospitals with ICP monitoring utilization of more than $80 \%$ were assigned to the high group, and the remaining 367 patients were assigned to the low group.

Table 1 shows the clinical characteristics and outcomes of the study participants. The median age in this study population was 65 years (interquartile [IQR] 46-77), and 282 (66\%) participants were male. Six months after the TBI, 137 (32\%) patients had a favorable functional outcome. There were significant differences between the high and the low groups with regard to the distribution of the Marshall CT classification and the ISS. Moreover, compared with the low group, the high group was more frequently treated with ICP monitoring and at the university hospitals, and had a high proportion of favorable functional outcome.

The results of the multiple logistic regression analyses are summarized in Table 2. The model 1 revealed an association between the high group and increased favorable functional outcome (adjusted odds ratio

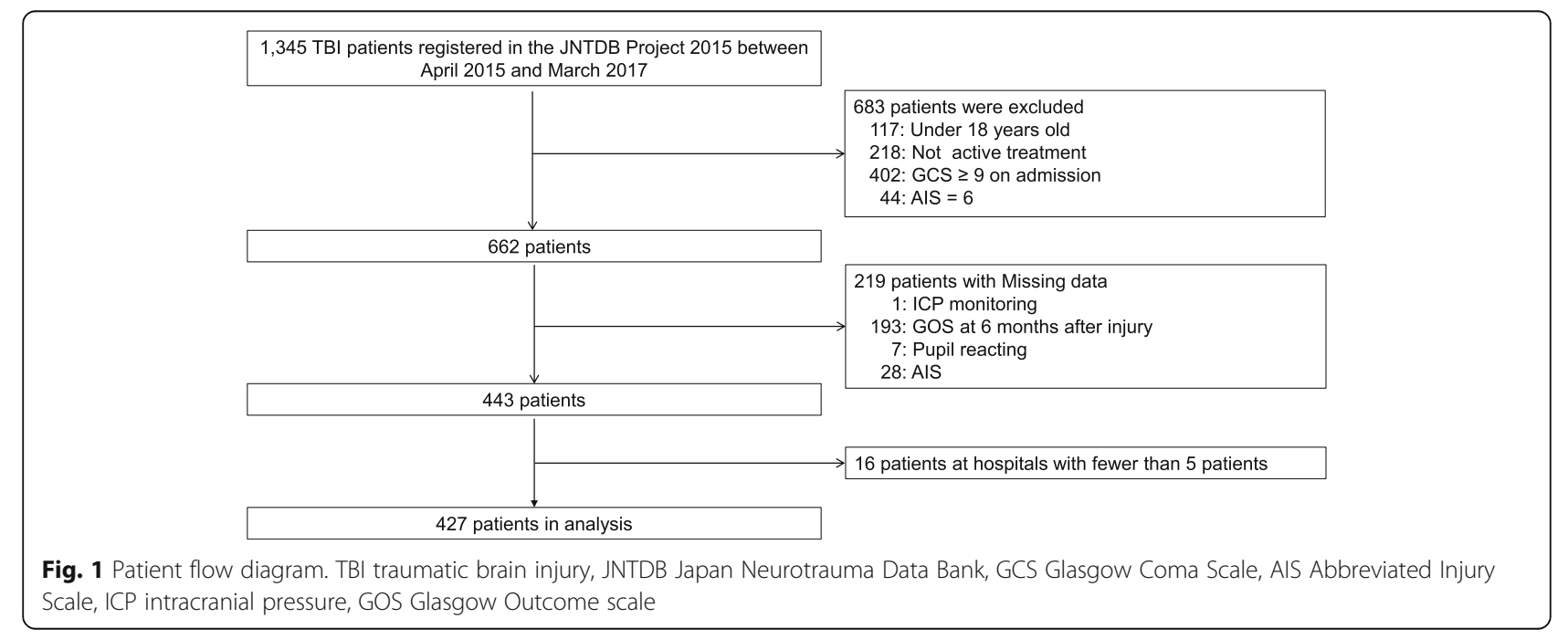




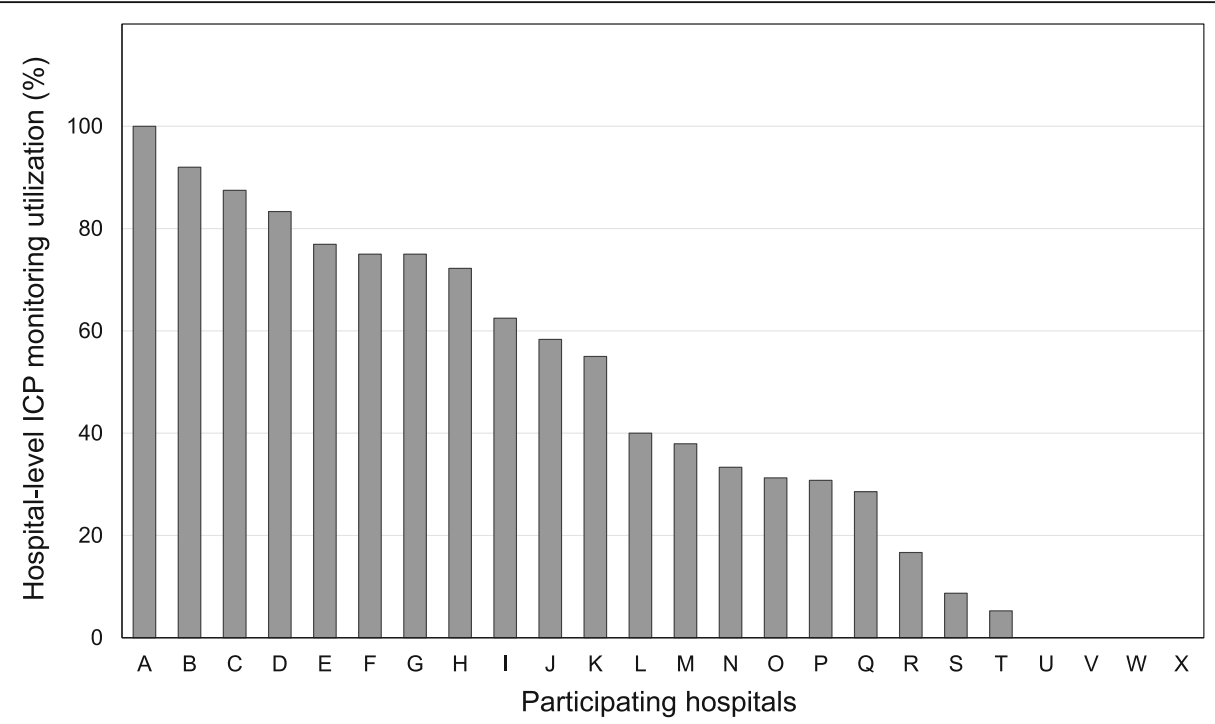

Fig. 2 Inter-hospital variation of intracranial pressure monitoring utilization

[OR]: 2.36; 95\% confidence interval (CI): 1.17-4.76; $p=0.016)$. Moreover, this association was observed in the model 2 that was adjusted for the age, GCS motor score, pupillary reflex, hypotension on admission, Marshall CT classification, ISS, and hospital type (adjusted OR: $2.30 ; 95 \%$ CI: $1.07-4.92 ; p=0.033$ ).

The intergroup analysis based on the subclassification by hospital-level ICP monitoring utilization showed that, with regard to the $0-19 \%$ group, only the $80-100 \%$ group was significantly associated with increased favorable functional outcome - both in the model 1 and model 2 multiple logistic regression analyses (Fig. 3).

The results of patient-level intergroup comparison of the ICP $(+)$ and ICP (-) groups are shown in Table 3, and showed significant differences in the pupillary reflex, hypotension, the distribution of the Marshall CT classification, hospital type, and the distribution of GOS at 6 months after injury. After adjusting the covariates, neither the model 1 nor model 2 multiple logistic regression analyses showed an association between patient-level ICP monitoring utilization and better functional outcome (Table 4).

\section{Discussion}

To our knowledge, this is the first study to assess the association of hospital-level ICP monitoring utilization with functional outcome in patients with severe TBI. In this study, we found that treatment at hospitals with ICP monitoring utilization of more than $80 \%$ was significantly associated with better functional outcome at 6 months after injury in patients with severe TBI. However, the patient-level
ICP monitoring utilization was unrelated to functional outcome.

Two previous studies assessed the association between hospital-level ICP monitoring utilization and in-hospital mortality [5, 9]. Alali et al. showed that patients with severe TBI at hospitals with ICP monitoring utilization rates of more than $16.1 \%$ had significantly improved outcomes than those at hospitals with rates of less than $8.3 \%$ [5]. This threshold was considerably lower than our threshold of $80 \%$, and the discrepancy may be attributable to the difference in the outcomes. Whereas we set the 6-month functional outcome as the primary outcome, Alali et al. used an entirely different metric - in-hospital mortality. In general, it is more difficult to improve functional outcome than to decrease mortality, as several previous studies on neurocritical care have suggested [21, 22]; thus, it may be reasonable that the clinical threshold of better functional outcome is higher than that of decreased mortality. A study by Dawes and colleagues did not find an association of high hospital-level ICP monitoring utilization with improved outcome [9]. We excluded patients with any Abbreviated Injury Scale score of 6, who were not expected to survive; however, Dawes et al. did not exclude their patients based on this criteria and, therefore, the patients in their study were considered to be too severely ill to obtain benefits from hospitals with high hospital-level ICP monitoring utilization.

We did not evaluate the factors at the hospitals with high ICP monitoring utilization to assess which worked well on the functional outcome. The patientlevel ICP monitoring utilization may explain this 
Table 1 Baseline characteristics and outcomes, Treatment at the hospitals with high ICP monitoring utilization vs. low utilization

\begin{tabular}{|c|c|c|c|c|c|}
\hline Variables & Overall $n=427$ & High group $n=60$ & Low group $n=367$ & $P$ value & Missing data \\
\hline Age, years, median [IQR] & $65[46,77]$ & $65[43,78]$ & $66[47,77]$ & 0.755 & 0 \\
\hline Male sex, n (\%) & $282(66)$ & $36(60)$ & $246(67)$ & 0.305 & 0 \\
\hline Prescribing anticoagulant drugs, n (\%) & $19(4.4)$ & $2(3.3)$ & $17(4.6)$ & 1 & 0 \\
\hline Prescribing antiplatelet drugs, n (\%) & $36(8.4)$ & $7(11.7)$ & $29(7.9)$ & 0.319 & 0 \\
\hline Glasgow Coma Scale score, median [IQR] & & & & & 0 \\
\hline Overall score & $6[3,7]$ & $6[3,7]$ & $6[3,7]$ & 0.305 & \\
\hline Motor score & $3[1,4]$ & $4[1,5]$ & $3[1,4]$ & 0.288 & \\
\hline Pupillary reflex, n (\%) & & & & 0.334 & 0 \\
\hline None & $110(26)$ & $13(22)$ & $97(26)$ & & \\
\hline One & $91(21)$ & $17(28)$ & $74(20)$ & & \\
\hline Both & $226(53)$ & $30(50)$ & $196(53)$ & & \\
\hline Hypotension, n (\%) & $60(14)$ & $7(12)$ & $53(14)$ & 0.69 & 0 \\
\hline Body temperature, ${ }^{\circ} \mathrm{C}$, median [IQR] & $36.2[35.8,36.8]$ & $36.2[35.9,36.9]$ & $36.2[35.8,36.8]$ & 0.367 & 34 \\
\hline Marshall CT classification, n (\%) & & & & 0.002 & 0 \\
\hline diffuse injury I & $9(2.1)$ & $1(1.7)$ & $8(2.2)$ & & \\
\hline diffuse injury ॥ & $96(22.5)$ & $12(20.0)$ & $84(22.9)$ & & \\
\hline diffuse injury III & $48(11.2)$ & $3(5.0)$ & $45(12.3)$ & & \\
\hline diffuse injury IV & $11(2.6)$ & $2(3.3)$ & $9(2.5)$ & & \\
\hline evacuated mass & $175(41.0)$ & $38(63.3)$ & $137(37.3)$ & & \\
\hline non-evacuated mass & $88(20.6)$ & $4(6.7)$ & $84(22.9)$ & & \\
\hline Injury Severity Score, median [IQR] & $25[25,35]$ & $25[18,29]$ & $25[18,29]$ & 0.045 & 0 \\
\hline Isolated traumatic brain injury, n (\%) & $173(41)$ & $28(47)$ & $145(40)$ & 0.322 & 0 \\
\hline Cause of injury, n (\%) & & & & 0.344 & 2 \\
\hline Motor vehicle & $27(6.3)$ & $26(7.1)$ & $1(1.7)$ & & \\
\hline Motorcycle & $53(12.4)$ & $45(12.3)$ & $8(13.6)$ & & \\
\hline Bicycle & $34(8.0)$ & $28(7.7)$ & $6(10.2)$ & & \\
\hline Pedestrian & $90(21.1)$ & $82(22.4)$ & $8(13.6)$ & & \\
\hline High-level fall & $115(27.0)$ & $95(26.0)$ & $20(33.9)$ & & \\
\hline Ground-level fall & 79 (18.5) & $68(18.6)$ & $11(18.6)$ & & \\
\hline Others & $18(4.2)$ & $22(6.0)$ & $5(8.5)$ & & \\
\hline Treatment with ICP monitoring, $\mathrm{n}(\%)$ & $192(45)$ & $55(92)$ & $137(37)$ & $<0.001$ & 0 \\
\hline Treatment at university hospitals, n (\%) & $257(60)$ & $45(75)$ & $212(58)$ & 0.015 & 0 \\
\hline GOS at 6 months after injury, n (\%) & & & & 0.074 & 0 \\
\hline Good recovery & 75 (17.6) & $12(20.0)$ & $63(17.2)$ & & \\
\hline Moderate disability & $62(14.5)$ & $15(25.0)$ & $47(12.8)$ & & \\
\hline Severe disability & $40(9.4)$ & $7(11.7)$ & $33(9.0)$ & & \\
\hline Vegetable state & $41(9.6)$ & $4(6.7)$ & $37(10.1)$ & & \\
\hline Death & $209(48.9)$ & $22(36.7)$ & $187(51.0)$ & & \\
\hline Favorable outcome at 6 months after injury, n (\%) & $137(32)$ & $27(45)$ & $110(30)$ & 0.025 & 0 \\
\hline
\end{tabular}

IQR interquartile range, $C T$ computed tomography, ICP intracranial pressure, GOS Glasgow Outcome Scale

association, because the high group was more frequently treated with ICP monitoring than the low group ( $92 \%$ vs $37 \%, p<0.001)$. However, our patient- level analysis and previous studies did not support this suspicion. Furthermore, ICP monitoring is not a therapeutic intervention but is merely a monitoring system. 
Table 2 Association between hospital-level ICP monitoring utilization and favorable functional outcome

\begin{tabular}{|c|c|c|c|c|}
\hline \multirow[t]{2}{*}{ Variables } & \multicolumn{2}{|l|}{ Model 1} & \multicolumn{2}{|l|}{ Model 2} \\
\hline & Adjusted OR (95\% CI) & $P$ value & Adjusted OR (95\% Cl) & $P$ value \\
\hline Higher hospital-level ICP monitoring utilization & $2.36(1.17-4.76)$ & 0.016 & $2.30(1.07-4.92)$ & 0.033 \\
\hline Age, per year & $0.94(0.93-0.96)$ & $<0.001$ & $0.93(0.92-0.95)$ & $<0.001$ \\
\hline Glasgow Coma Scale Motor score, per point & $1.50(1.25-1.80)$ & $<0.001$ & $1.40(1.16-1.69)$ & $<0.001$ \\
\hline \multicolumn{5}{|l|}{ Pupillary reflex } \\
\hline None & Reference & - & Reference & - \\
\hline One & $5.27(2.11-13.20)$ & $<0.001$ & $4.53(1.72-11.90)$ & 0.002 \\
\hline Both & $10.00(4.19-24.00)$ & $<0.001$ & $7.28(2.86-18.50)$ & $<0.001$ \\
\hline Hypotension on admission & - & - & $0.29(0.09-0.96)$ & 0.042 \\
\hline Marshall CT classification & - & - & & \\
\hline Diffuse injury I & - & - & Reference & - \\
\hline Diffuse injury ॥ & - & - & $0.16(0.00-5.12)$ & 0.300 \\
\hline Diffuse injury III & - & - & $0.05(0.00-1.80)$ & 0.100 \\
\hline Diffuse injury IV & - & - & $0.10(0.00-4.37)$ & 0.230 \\
\hline Evacuated mass & - & - & $0.10(0.00-3.08)$ & 0.190 \\
\hline Non-evacuated mass & - & - & $0.12(0.00-3.76)$ & 0.220 \\
\hline Injury Severity Score, per point & - & - & $0.95(0.93-0.98)$ & $<0.001$ \\
\hline Treatment at university hospitals & - & - & $0.97(0.55-1.69)$ & 0.902 \\
\hline
\end{tabular}

$O R$ odds ratio, $C l$ confidence interval, ICP intracranial pressure, $C T$ computed tomography

Therefore, whether patients can obtain net benefits from ICP monitoring apparently depends on whether their physicians can effectively utilize ICP information in a timely manner. Physicians at hospitals with high ICP monitoring utilization may have better-quality opportunities to develop their skills in optimizing ICP monitoring. Perhaps, it is for this reason that patients treated at hospitals with high ICP monitoring utilization had better functional outcomes. Further investigation is needed to validate the preliminary findings of this study and to validate the role of ICP monitoring utilization in the severe TBI patient population.

The present study has several limitations. First, this was a post hoc analysis of a prospective multicenter cohort study. We conducted multiple logistic regression analyses that were adjusted for clinically important factors; however, selection bias and uncontrolled confounding variables may have influenced the results due to the observational study design. Second, we excluded 219 of the 662 potentially eligible patients due to missing data for important variables (Fig. 1). Thus, the baseline characteristics of the patients included in the final analysis were different from those of the excluded patients (Table E1, web-only appendices)), which may have led to some selection biases. Third, there were no specific management protocols for severe TBI in the JNTDB study, and treatment strategies depended crucially on each institution; thus,

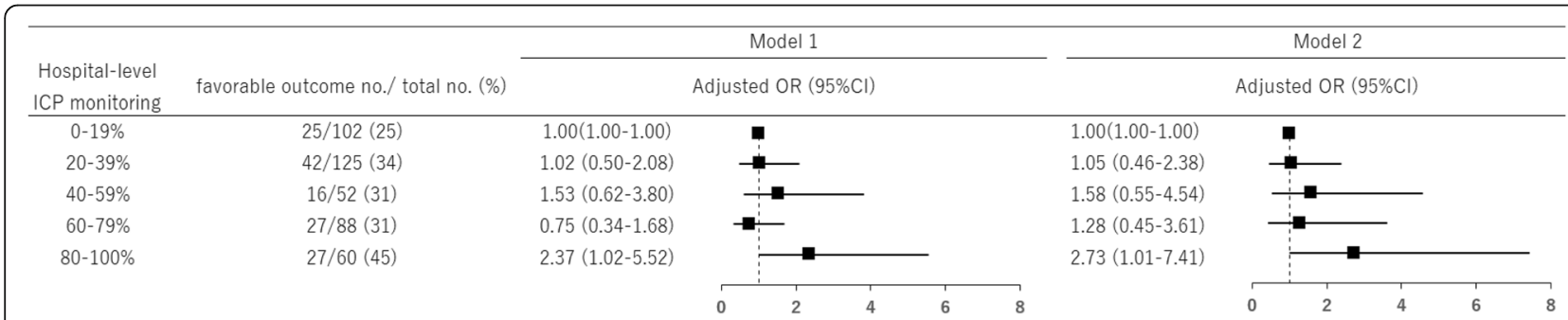

Fig. 3 Association between the subclassification of hospital-level ICP monitoring utilization and favorable functional outcome. ICP intracranial pressure, OR odds ratio, Cl confidence interval. Model 1 was adjusted for age, Glasgow Coma Scale Motor score, and pupillary reflex. Model 2 was adjusted for age, Glasgow Coma Scale Motor score, pupillary reflex, hypotension an admission, Marshall CT classification, Injury Severity Score, and hospital type 
Table 3 Baseline characteristics and outcomes, treatment with vs. without ICP monitoring

\begin{tabular}{|c|c|c|c|c|c|}
\hline Variables & Overall $n=427$ & ICP $(+)$ group $n=192$ & ICP $(-)$ group $n=235$ & $P$ value & Missing data \\
\hline Age, years, median [IQR] & $65[46,77]$ & $65[45,76]$ & $66[50,79]$ & 0.173 & 0 \\
\hline Male sex, n (\%) & $282(66)$ & $121(63)$ & $161(69)$ & 0.259 & 0 \\
\hline Prescribing anticoagulant drugs, n (\%) & $19(4.4)$ & $7(3.6)$ & $12(5.1)$ & 0.492 & 0 \\
\hline Prescribing antiplatelet dugs, n (\%) & $36(8.4)$ & $17(8.9)$ & $19(8.1)$ & 0.861 & 0 \\
\hline Glasgow Coma Scale score, median [IQR] & & & & & 0 \\
\hline Overall score & $6[3,7]$ & $6[3,7]$ & $6[3,7]$ & 0.566 & \\
\hline Motor score & $3[1,4]$ & $3[1,4]$ & $4[1,4]$. & 0.614 & \\
\hline Pupillary reflex, n (\%) & & & & 0.002 & 0 \\
\hline None & $110(26)$ & $39(20)$ & $71(30)$ & & \\
\hline One & $91(21)$ & $55(29)$ & $36(15)$ & & \\
\hline Both & $226(53)$ & $98(51)$ & $128(55)$ & & \\
\hline Hypotension, n (\%) & $60(14)$ & $16(8)$ & $44(19)$ & 0.002 & 0 \\
\hline Body temperature, ${ }^{\circ} \mathrm{C}$, median $[\mathrm{QQR}]$ & $36.2[35.8,36.8]$ & $36.2[35.8,36.8]$ & $36.2[35.8,36.8]$ & 0.876 & 34 \\
\hline Marshall CT classification, n (\%) & & & & 0.001 & 0 \\
\hline diffuse injury I & $9(2.1)$ & $2(1.0)$ & $7(3.0)$ & & \\
\hline diffuse injury ॥ & $96(22.5)$ & $29(15.1)$ & $67(28.5)$ & & \\
\hline diffuse injury III & $48(11.2)$ & $24(12.5)$ & $24(10.2)$ & & \\
\hline diffuse injury IV & $11(2.6)$ & $5(2.6)$ & $6(2.6)$ & & \\
\hline evacuated mass & $175(41.0)$ & $98(51.0)$ & $77(32.8)$ & & \\
\hline non-evacuated mass & $88(20.6)$ & $34(17.7)$ & $54(23.0)$ & & \\
\hline Injury Severity Score, median [IQR] & $25[25,35]$ & $26[25,34]$ & $25[22,37]$ & 0.441 & 0 \\
\hline Isolated traumatic brain injury, n (\%) & $173(41)$ & $77(40)$ & $96(41)$ & 0.921 & 0 \\
\hline Cause of injury, n (\%) & & & & 0.703 & 2 \\
\hline Motor vehicle & $27(6.3)$ & $12(6.3)$ & $15(6.4)$ & & \\
\hline Motorcycle & $53(12.4)$ & $25(13.1)$ & $28(12.0)$ & & \\
\hline Bicycle & $34(8.0)$ & $42(22.0)$ & $48(20.5)$ & & \\
\hline Pedestrian & $90(21.1)$ & $17(8.9)$ & $17(7.3)$ & & \\
\hline High-level fall & $115(27.0)$ & $54(28.3)$ & $61(26.1)$ & & \\
\hline Ground-level fall & $79(18.5)$ & $28(14.7)$ & $51(21.8)$ & & \\
\hline Others & $18(4.2)$ & $13(6.8)$ & $14(6.0)$ & & \\
\hline Treatment at university hospitals, n (\%) & $257(60)$ & $146(76.0)$ & $111(47.2)$ & $<0.001$ & 0 \\
\hline GOS at 6 months after injury, n (\%) & & & & 0.001 & 0 \\
\hline Good recovery & 75 (17.6) & $28(14.6)$ & $47(20.0)$ & & \\
\hline Moderate disability & $62(14.5)$ & 368 (18.8) & $26(11.1)$ & & \\
\hline Severe disability & $40(9.4)$ & $21(10.9)$ & $19(8.1)$ & & \\
\hline Vegetable state & $41(9.6)$ & $27(14.1)$ & $14(6.0)$ & & \\
\hline Death & $209(48.9)$ & $80(41.7)$ & $129(54.9)$ & & \\
\hline Favorable outcome at 6 months after injury, n (\%) & $137(32)$ & $64(33)$ & $73(31)$ & 0.677 & 0 \\
\hline
\end{tabular}

$I Q R$ interquartile range, $C T$ computed tomography, ICP intracranial pressure, GOS Glasgow Outcome Scale

this may have led to additional biases. Furthermore, we did not consider hospital-level factors other than the utilization rate of ICP monitoring and hospital type (university or non-university). Finally, there are two possible generalization problems: (1) as these results were derived from the JNTDB Project 2015 that was conducted in the Japanese population, the findings may not be generalizable to other countries and (2) the reason for the distribution trend of ICP monitoring utilization across the study centers was unclear, and 
Table 4 Association between patient-level ICP monitoring utilization and favorable functional outcome

\begin{tabular}{|c|c|c|c|c|}
\hline \multirow[t]{2}{*}{ Variables } & \multicolumn{2}{|l|}{ Model 1} & \multicolumn{2}{|l|}{ Model 2} \\
\hline & Adjusted OR (95\% CI) & $P$ value & Adjusted OR $(95 \% \mathrm{Cl})$ & $P$ value \\
\hline Treatment with ICP monitoring & $0.96(0.57-1.59)$ & 0.863 & $1.08(0.60-1.94)$ & 0.791 \\
\hline Age, per year & $0.94(0.93-0.96)$ & $<0.001$ & $0.94(0.92-0.95)$ & $<0.001$ \\
\hline Glasgow Coma Scale Motor score, per point & $1.52(1.27-1.82)$ & $<0.001$ & $1.41(1.16-1.70)$ & $<0.001$ \\
\hline \multicolumn{5}{|l|}{ Pupillary reflex } \\
\hline None & Reference & - & Reference & - \\
\hline One & $5.40(2.15-13.60)$ & $<0.001$ & $4.74(1.79-12.50)$ & 0.002 \\
\hline Both & $9.59(4.05-22.70)$ & $<0.001$ & $7.26(2.89-18.30)$ & $<0.001$ \\
\hline Hypotension on admission & - & - & $0.31(0.09-1.03)$ & 0.056 \\
\hline Marshall CT classification & - & - & & \\
\hline Diffuse injury I & - & - & Reference & - \\
\hline Diffuse injury ॥ & - & - & $0.16(0.01-4.81)$ & 0.294 \\
\hline Diffuse injury III & - & - & $0.05(0.00-1.65)$ & 0.094 \\
\hline Diffuse injury IV & - & - & $0.11(0.00-4.44)$ & 0.239 \\
\hline Evacuated mass & - & - & $0.11(0.00-3.09)$ & 0.192 \\
\hline Non-evacuated mass & - & - & $0.11(0.00-3.29)$ & 0.203 \\
\hline Injury Severity Score, per point & - & - & $0.95(0.93-0.98)$ & $<0.001$ \\
\hline Treatment at university hospitals & - & - & $1.01(0.57-1.79)$ & 0.975 \\
\hline
\end{tabular}

$O R$ odds ratio, $C l$ confidence interval, ICP intracranial pressure, $C T$ computed tomography

therefore, it is unknown whether the generalization of the results to other settings would be feasible.

\section{Conclusions}

In summary, treatment at hospitals with high ICP monitoring utilization for severely ill patients with TBI was associated with better functional outcome.

\section{Supplementary Information}

The online version contains supplementary material available at https://doi. org/10.1186/s13049-020-00825-7.

Additional file 1: Additional Table 1. Baseline characteristics, inclusion vs. exclusion group

\section{Abbreviations}

TBI: Traumatic brain injury; GCS: Glasgow Coma Scale; ICP: Intracranial pressure; JNTDB: Japan Neurotrauma Data Bank; GOS: Glasgow Outcome Scale; CT: Computed Tomography; ISS: Injury Severity Score; IMPA CT: International Mission for Prognosis and Analysis of Clinical Trials

\section{Acknowledgements}

We are grateful to all investigators and institutions of the JNTDB Project 2015: Department of Neurosurgery, Sapporo Medical University; Department of Neurosurgery, Aomori Prefectural Central Hospital; Department of Neurosurgery, Sendai City Hospital; Department of Neurosurgery, National Hospital Organization, Shinshu Ueda Medical Center; Department of Neurosurgery, Chiba Emergency Medical Center; Department of Neurological Surgery, Chiba University Graduate School of Medicine; Department of Neurosurgery, Kimitsu Chuo Hospital; Emergency and Critical Care Center, Jikei University Kashiwa Hospital; Department of Emergency, Disaster and Critical Care Medicine, Showa University; Trauma and Critical Care Center, Teikyo University School of Medicine; Department of Emergency and Critical Care Medicine, Nippon Medical School; Department of Neurological Surgery,
Nihon University School of Medicine; Division of Neurosurgery, National Hospital Organization Disaster Medical Center; Department of Neurosurgery, National Defense Medical College; Department of Neurosurgery, Yokohama City University Medical Center; Department of Neurosurgery, St. Marianna University School of Medicine; Department of Neurosurgery, Tokai University School of Medicine; 2nd Department of Neurosurgery, Toho University Ohashi Medical Center; Department of Emergency and Critical Care Medicine, Nippon Medical School Tama-Nagayama Hospital; Department of Neurosurgery, Tokyo Medical and Dental University; Department of Neurosurgery and Restorative Neuroscience, Graduate School of Medical Sciences, Nagoya City University; Emergency and critical care medicine, Saiseikai Shiga Hospital; Department of Neurosurgery, Kyoto Kujo Hospital; Department of Neurosurgery, Nara Medical University; Osaka Mishima Emergency Critical Care Center; Department of Neurosurgery, Hyogo Prefectural Kakogawa Medical Center; Emergency Medical Center, Kagawa University Hospital; Department of Neurosurgery, Yamaguchi University Graduate School of Medicine; Department of Emergency Medicine, lizuka Hospital, Department of Neurosurgery; Kurume University School of Medicine; Department of Neurosurgery, Faculty of Medicine, Fukuoka University; Department of Neurosurgery, Kumamoto Red Cross Hospital.

\section{Authors' contributions}

TO were responsible for the article and drafted and revised the manuscript. KK and YK helped to draft the manuscript. All authors have read and approved the final manuscript and take full responsibility for all aspects of the study.

\section{Funding}

The Japan Neurotrauma Data Bank (JNTDB) Project 2015 was supported by the Japan Society of Neurotraumatology. The authors received no financial support for the current post hoc analysis.

\section{Availability of data and materials}

The datasets used in the current study are available from the corresponding author on reasonable request.

\section{Ethics approval and consent to participate}

The protocol was approved by the Institutional Review Board of each participating hospital. 


\section{Consent for publication}

Not applicable.

\section{Competing interests}

The authors declare that they have no conflicts of interest.

Received: 23 July 2020 Accepted: 13 December 2020

Published online: 06 January 2021

\section{References}

1. Maas AIR, Menon DK, Adelson PD, Andelic N, Bell MJ, Belli A, et al. Traumatic brain injury: integrated approaches to improve prevention, clinical care, and research. Lancet Neurol. 2017;16:987-1048.

2. Rosenfeld JV, Maas Al, Bragge P, Morganti-Kossmann MC, Manley GT, Gruen RL. Early management of severe traumatic brain injury. Lancet. 2012;380: 1088-98.

3. Smith M. Monitoring intracranial pressure in traumatic brain injury. Anesth Analg. 2008;106:240-8

4. Kinoshita K. Traumatic brain injury: pathophysiology for neurocritical care. Intensive Care. 2016;4:29.

5. Alali AS, Fowler RA, Mainprize TG, Scales DC, Kiss A, de Mestral C, et al. Intracranial pressure monitoring in severe traumatic brain injury: results from the American College of Surgeons trauma quality improvement program. J Neurotrauma. 2013;30:1737-46.

6. Shen L, Wang Z, Su Z, Qiu S, Xu J, Zhou Y, et al. Effects of intracranial pressure monitoring on mortality in patients with severe traumatic brain injury: a meta-analysis. PLoS One. 2016;11:e0168901.

7. Chesnut RM, Temkin N, Carney N, Dikmen S, Rondina C, Videtta W, et al. A trial of intracranial-pressure monitoring in traumatic brain injury. N Engl J Med. 2012;367:2471-81.

8. Agrawal D, Raghavendran K, Schaubel DE, Mishra MC, Rajajee V. A propensity score analysis of the Impact of invasive intracranial pressure monitoring on outcomes after severe traumatic brain injury. J Neurotrauma. 2016:33:853-8.

9. Dawes AJ, Sacks GD, Cryer HG, Gruen JP, Preston C, Gorospe D, et al. Compliance with evidence-based guidelines and Interhospital variation in mortality for patients with severe traumatic brain injury. JAMA Surg. 2015; 150:965-72.

10. Suehiro E, Fujiyama $Y$, Koizumi $H$, Suzuki M. Directions for use of intracranial pressure monitoring in treatment of severe traumatic brain injury using data from the Japan Neurotrauma data Bank. J Neurotrauma. 2017;34:2230-4.

11. Suehiro E, Fujiyama Y, Kiyohira M, Haji K, Ishihara H, Nomura S, et al. Risk of deterioration of geriatric traumatic brain injury in patients treated with antithrombotic drugs. World Neurosurg. 2019;127:e1221-e7.

12. Nakamura N, Yamaura A, Shigemori M, Ogawa T, Tokutomi T, Ono J, et al. Final report of the Japan Neurotrauma Data Bank project 1998-2001: 1,002 cases of traumatic brain injury. Neurol Med Chir. 2006;46:567-74.

13. Yokobori S, Yamaguchi M, Igarashi $Y$, Hironaka $K$, Onda H, Kuwamoto K, et al. Outcome and Refractory Factor of Intensive Treatment for Geriatric Traumatic Brain Injury: Analysis of 1165 Cases Registered in the Japan Neurotrauma Data Bank. World Neurosurg. 2016;86:127-33.e1.

14. Hesdorffer DC, Ghajar J. Marked improvement in adherence to traumatic brain injury guidelines in United States trauma centers. J Trauma. 2007;63: 841-7 discussion 7-8.

15. Gerber LM, Chiu YL, Carney N, Hartl R, Ghajar J. Marked reduction in mortality in patients with severe traumatic brain injury. J Neurosurg. 2013; 119:1583-90.

16. Jennett $B$, Bond M. Assessment of outcome after severe brain damage. Lancet. 1975:1:480-4.

17. Murray GD, Butcher I, McHugh GS, Lu J, Mushkudiani NA, Maas Al, et al. Multivariable prognostic analysis in traumatic brain injury: results from the IMPACT study. J Neurotrauma. 2007:24:329-37.

18. Steyerberg EW, Mushkudiani N, Perel P, Butcher I, Lu J, McHugh GS, et al. Predicting outcome after traumatic brain injury: development and international validation of prognostic scores based on admission characteristics. PLoS Med. 2008:5:e165 discussion e.

19. Panczykowski DM, Puccio AM, Scruggs BJ, Bauer JS, Hricik AJ, Beers SR, et al. Prospective independent validation of IMPACT modeling as a prognostic tool in severe traumatic brain injury. J Neurotrauma. 2012;29:47-52.
20. Kanda Y. Investigation of the freely available easy-to-use software 'EZR' for medical statistics. Bone Marrow Transplant. 2013:48:452-8.

21. Hutchinson PJ, Kolias AG, Timofeev IS, Corteen EA, Czosnyka M, Timothy J, et al. Trial of Decompressive Craniectomy for traumatic intracranial hypertension. N Engl J Med. 2016;375:1119-30.

22. Sprigg N, Flaherty K, Appleton JP, Al-Shahi Salman R, Bereczki D, Beridze M, et al. Tranexamic acid for hyperacute primary IntraCerebral Haemorrhage (TICH-2): an international randomised, placebo-controlled, phase 3 superiority trial. Lancet. 2018;391:2107-15.

\section{Publisher's Note}

Springer Nature remains neutral with regard to jurisdictional claims in published maps and institutional affiliations.
Ready to submit your research? Choose BMC and benefit from:

- fast, convenient online submission

- thorough peer review by experienced researchers in your field

- rapid publication on acceptance

- support for research data, including large and complex data types

- gold Open Access which fosters wider collaboration and increased citations

- maximum visibility for your research: over $100 \mathrm{M}$ website views per year

At BMC, research is always in progress.

Learn more biomedcentral.com/submissions 